\title{
Metachronous endobronchial metastasis from uterine cervical adenocarcinoma
}

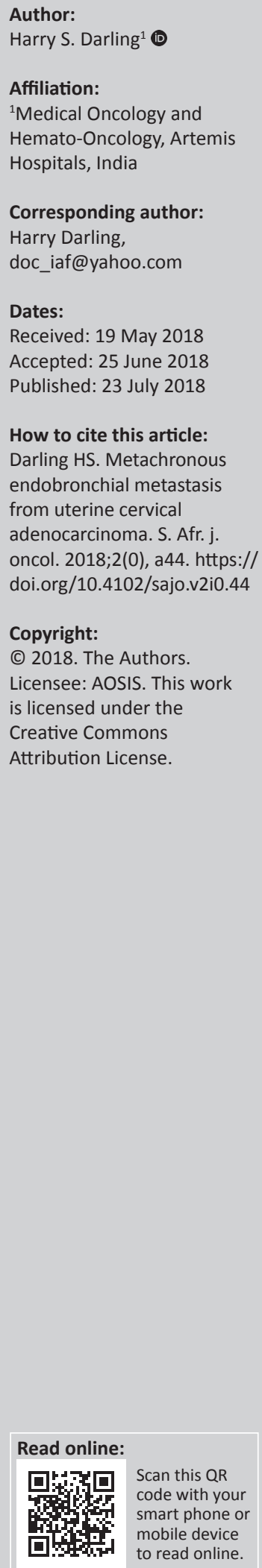

\begin{abstract}
Malignant involvement of the lung can be primary or metastatic. Although primary lung cancer may be endobronchial, parenchymal or both, metastases to the lung are generally parenchymal. However, less commonly, they may be endobronchial. The lungs are the most common site of metastases among all the malignancies. Common extrapulmonary malignancies causing endobronchial metastases (EBMs) are breast, colorectal and kidney. EBMs from uterine cervical cancer are very rare. Although there are a few case reports in the medical literature describing EBM from cervical cancer, none are from adenocarcinoma histology. In this study, we report a case of EBM which on histopathological examination and immunohistochemistry were found to be from a prior adenocarcinoma cervix. Differentiating EBM from bronchogenic carcinoma and other more common extrapulmonary solid organ malignancies may present a clinico-pathological and radiological challenge.
\end{abstract}

\section{Introduction}

Cancer of the uterine cervix is the third most common cancer in women worldwide. ${ }^{1,2}$ However, in women in developing nations, it is the second most common malignancy and cause of cancer mortality. ${ }^{3}$ Squamous cell carcinoma of the uterine cervix is the most common histology (80\%) followed by adenocarcinoma (20\%). ${ }^{4}$ Most cases present as early or locally advanced stages; metastases to extrapelvic organs are less common. Pulmonary metastases have been reported in $3.1 \%-8.2 \%$ of patients with uterine cervical cancer. ${ }^{5}$ As most of the pulmonary metastases from extrapulmonary solid organs are parenchymal, same holds true for metastatic cervical cancer. Endobronchial metastases (EBMs) from cervical cancer have only occasionally been reported. ${ }^{5,6,7}$ Endobronchial metastases may mimic primary bronchogenic carcinoma clinicoradiologically as well as bronchoscopically. Additional immunohistochemical (IHC) staining, together with the specific clinical information, can help in differentiating the two pathologies. Here, we are reporting a case of an endobronchial tumour with adenocarcinoma histology in a patient who was treated for cervical cancer 1.5 years prior. Her background history and IHC helped establish the diagnosis of metastatic cervical adenocarcinoma. On literature search, we did not find any reported case of cervical cancer with adenocarcinoma histology metastasising endobronchially or the use of $\mathrm{p} 16^{\mathrm{INK} 4 \mathrm{a}} \mathrm{IHC}$ marker in an EBM to confirm the uterine cervix as primary site.

\section{Case report}

A 52-year-old woman was referred from a peripheral hospital with dry cough of 2 months duration and rapidly progressive dyspnoea more recently. Eighteen months back she was diagnosed as poorly differentiated adenocarcinoma cervix stage III B. She was treated with concurrent chemo-radiation followed by brachytherapy. Before the current presentation, there was no evidence of any disease progression. On examination, she had a tachycardia and was tachypnoeic and hypoxic ( $\mathrm{SpO}_{2} 80 \%$ room air). Her blood pressure was within normal limits. She had facial plethora, distended neck veins and hoarseness, suggestive of superior vena caval obstruction (SVCO). A plain radiograph (Figure 1) of the chest showed bilateral hilar adenopathy and collapse of right lower lobe. Contrast-enhanced computerised tomogram (CECT) chest and abdomen (Figures 2 and 3) showed a heterogeneously enhancing mass $7.5 \mathrm{~cm} \times 4 \mathrm{~cm} \times 6 \mathrm{~cm}$ arising from the left upper lobe (LUL) bronchus near its origin, causing subsegmental obstructive atelectasis of the LUL. There was bilateral hilar adenopathy, with complete obstructive collapse of right lower lobe (RLL), and bilateral multiple lung nodules. Fibreoptic bronchoscopy (FoB) revealed an endobronchial mass in LUL bronchus. Biopsy was consistent with poorly differentiated adenocarcinoma (Figure 4) which initially raised suspicion of bronchogenic carcinoma as second primary malignancy. Further, IHC staining (Figure 5) showed positive p16 ${ }^{\text {INK4a }}$ and negative thyroid transcription factor (TTF). On the basis of her clinical profile, pathology and IHC, she 


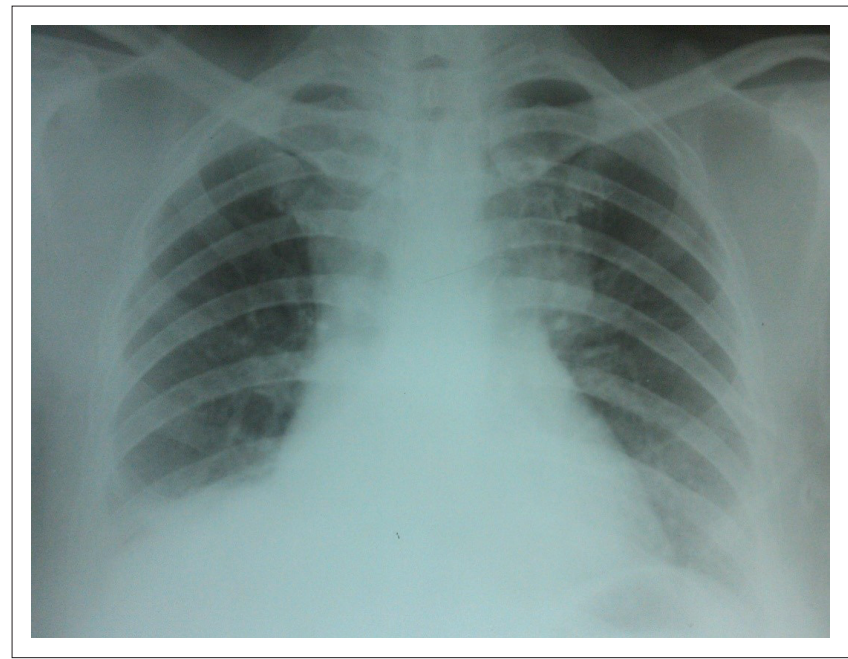

FIGURE 1: Chest X-ray (posteroanterior view) showing bilateral mediastinal adenopathy and right lower lobe (RLL) collapse.

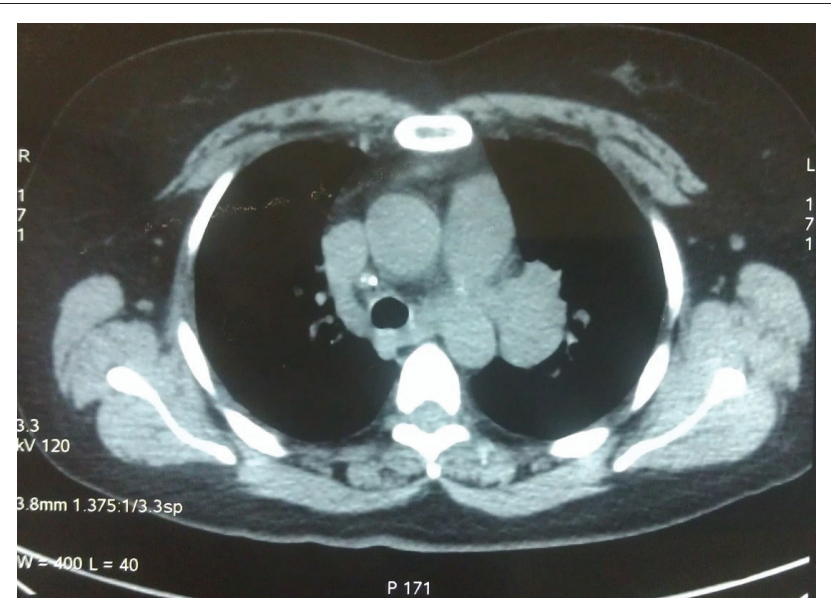

FIGURE 2: Computed tomography chest showing mass occluding left upper lobe (LUL) bronchus causing subsegmental collapse.

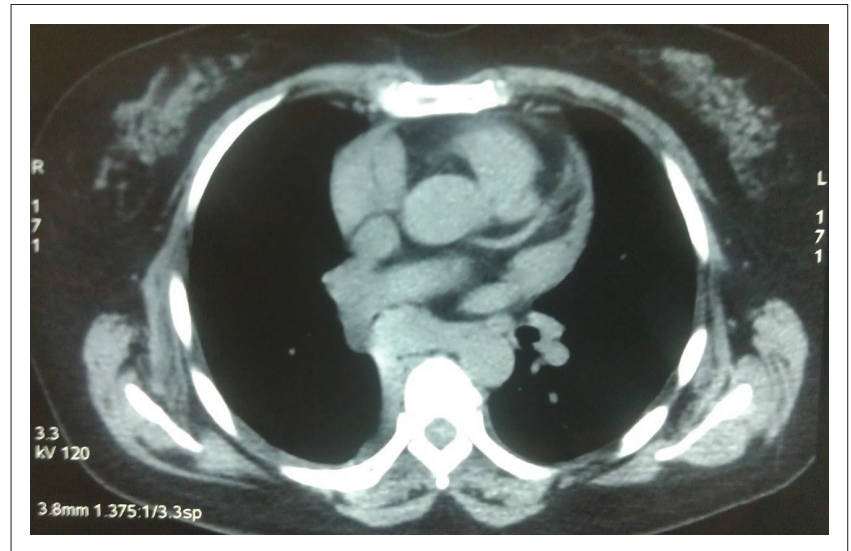

FIGURE 3: Computed tomography chest showing right hilar adenopathy and right lower lobe (RLL) collapse.

was diagnosed as metastatic endobronchial adenocarcinoma with uterine cervix as primary. Because of features of SVCO and severe dyspnoea, she was treated with palliative radiotherapy to the mediastinum and intravenous corticosteroids. After achieving symptomatic relief, she was started on palliative chemotherapy.

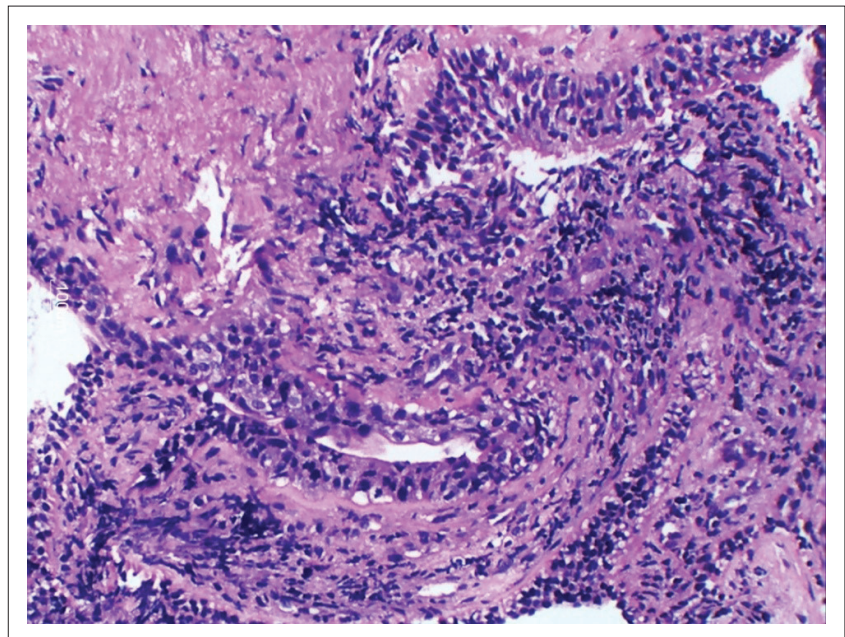

FIGURE 4: Bronchial biopsy (haemotoxylin and eosin, 400x) showing sheets of neoplastic cells with hyperchromatic nuclei and marked pleomorphism, embedded in a fibrous tissue. Attempted abortive glandular pattern is also seen, suggestive of poorly differentiated adenocarcinoma.

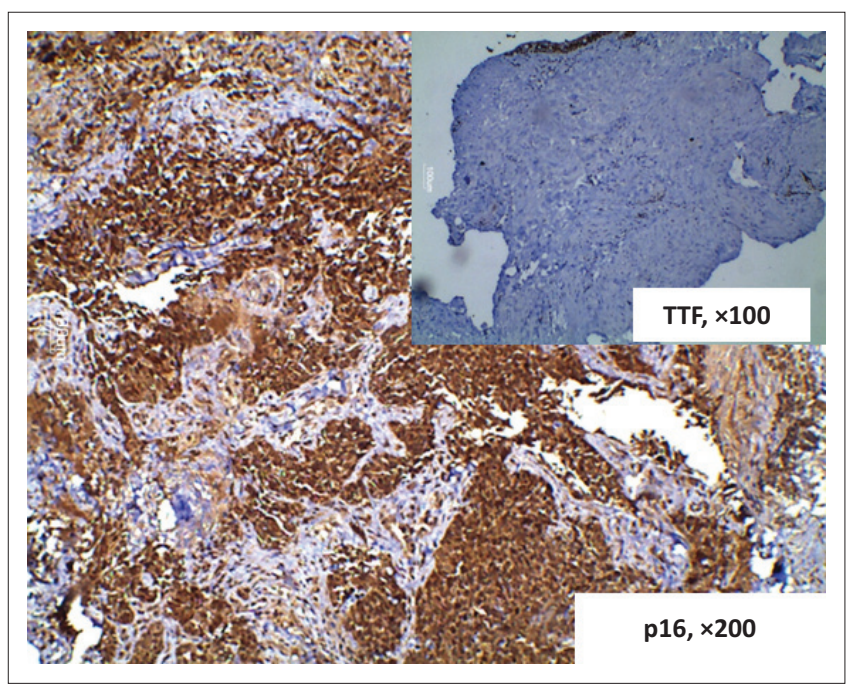

FIGURE 5: Immunohistochemical staining highlights the neoplastic cells to be positive for $\mathrm{p} 16$ and negative for thyroid transcription factor (TTF).

\section{Discussion}

While the incidence of pulmonary metastasis of nonpulmonary primary tumours is approximately $20 \%-50 \%$, that of EBM is very low, at most $5 \% .{ }^{6}$ In a case series of 18 cases of EBM, the most common primary tumour sites were colorectal (4), breast (3), renal (3), lymphoma (3), rhabdomyosarcoma (2), thyroid (1), bladder (1) and malignant melanoma (1). No case of cervical primary was identified. ${ }^{8}$ In contrast, Shepherd found 5 of 25 cases of EBM to be from primary uterine cervical cancer, all of which were of squamous histology. ${ }^{7}$ About $15 \%$ of uterine cervical cancer patients experience distant metastases, which may be synchronous or metachronous. ${ }^{9}$ The average interval of EBM to occur after the diagnosis of primary uterine cervical cancer is 5.4 years. ${ }^{10,11,12}$ In two cases reported by Yonsei et al., the interval was 3 and 2.2 years, respectively. ${ }^{6}$ In our case, the EBM was detected after an interval of 1.5 years, probably reflecting a more aggressive nature of the disease. 
Endobronchial metastases of non-pulmonary neoplasms and primary bronchogenic cancers can be difficult to differentiate. Routine histological examination, with additional IHC, is probably the best way to obtain an exact diagnosis. ${ }^{6}$ The radiological manifestations of EBM are quite diverse. Patients commonly present with hilar or mediastinal lymphadenopathy, atelectasis or even normal chest radiograph. Infrequently they may present with nonspecific pulmonary infiltrates and multiple pulmonary nodules. ${ }^{7,11,12,13}$ Fibreoptic bronchoscopy is the most important and simplest diagnostic tool and is essential for the diagnosis of endobronchial lesions, with a very high diagnostic accuracy for centrally located lesions. Also, in a known case of another non-pulmonary malignancy, the presence of persistent pulmonary symptoms warrants a FoB even with normal chest radiographic finding. Contrastenhanced computerised tomogram chest has high sensitivity in detecting and localising endobronchial lesions; hence, if FoB cannot be performed for whatever reason, a precise and accurate diagnosis can be made with CECT chest used in combination with sputum cytology and bronchial washes. ${ }^{6}$

The metastatic spread is presumed to be haematogenous, through the caval venous system, to the lung parenchyma and then the systemic circulation (bronchial arteries) and finally to emerge as EBM. Endobronchial metastasis seems to occur more commonly on the right side. Heitmiller et al. in their series reported that 20 of 25 cases were located to the right side, and both cases of Yonsei report were located on the right side. However, the significance of this predilection is not understood. ${ }^{6,10}$ Interestingly, in our case, EBM was on the left side.

Human papillomavirus (HPV) infection with high-risk serotypes is a significant risk factor in cervical cancer, being detectable in $99.7 \%$ cases. ${ }^{14}$ Among approximately 200 serotypes, only a small minority of high-risk serotype infected patients progress to cervical cancer after a long latency period. ${ }^{15} \mathrm{p} 16^{\mathrm{INK} 4 \mathrm{a}}$ is a tumour suppression protein which has been demonstrated as a useful marker for squamous and glandular epithelial dysplasia in the cervix. In cervical cancer, p16 ${ }^{\text {INK4a }}$ overexpression occurs because of functional inactivity of retinoblastoma gene (RB) by HPV E7 protein. In a study by Negri et al., p16 was detected immunohistochemically in all 26 cases of adenocarcinoma of the cervix uteri, including 18 invasive and 8 in situ carcinomas. ${ }^{16}$ Hence, p16 $6^{\text {INK4a }}$ is a useful marker for the diagnosis of squamous as well as adenocarcinoma of uterine cervix. ${ }^{17}$

Eun-Jung Ko et al. had reported the first case of EBM from the cervix, proven by HPV DNA genotyping. ${ }^{5}$ Our case appears to be the first where the diagnosis was proven by p16 ${ }^{\text {INK4a }}$ positivity on IHC.

\section{Conclusion}

Through this first reported case of adenocarcinoma cervix presenting metachronously as EBM, we demonstrated that p $16^{\text {INK4a }}$ IHC marker can be effectively used to differentiate a cervical carcinoma metastasis from a lung primary or other secondaries. Most of the EBMs from uterine cervical cancer reported in various studies have shown right-sided predominance, whereas in our case, EBM was on the left side. It must be emphasised that in a known case of extrapulmonary malignancy, even after a prolonged remission, the occurrence of new onset, persistent respiratory symptoms must be evaluated on imaging as well as bronchoscopically. Although the occurrence of a second malignancy is not uncommon, metastatic relapse of the first malignancy should be considered.

\section{Acknowledgements Competing interests}

The author declares that he has no financial or personal relationships that may have inappropriately influenced him in writing this article.

\section{References}

1. Parkin DM, Bray F, Ferlay J, Pisani P. Global cancer statistics. CA Cancer J Clin. 2011;61:69-90. https://doi.org/10.3322/caac.20107

2. Kamangar F, Dores GM, Anderson WF. Patterns of cancer incidence, mortality, and prevalence across five continents: Defining priorities to reduce cancer disparities in different geographic regions of the world. J Clinoncol. 2006;24:2137-2150. https://doi.org/10.1200/JCO.2005.05.2308

3. Jemel A, Bray F, Center MM, et al. Global cancer statistics. CA Cancer J Clin. 2005;55:79-7108.

4. Version 1.2014 @National Comprehensive Cancer Network, Inc. 2014.

5. Ko E-J, Hong W-J, Shin S-P, Shin S-Y, Heo J-H, Jeong H-C. Human papillomavirus type-16 positive endobronchial metastasis from uterine cervical cancer. Respirol Case Rep. 2014;2(1):10-12. https://doi.org/10.1002/rcr2.32

6. Kim YS, Chang J, Kim YS, et al. Endobronchialmetastasis of uterine cervix cancer: A two case reports \& a review of the literature. Yonsei Med J. 2002;43(4), 547-552.

7. Shepherd MP. Endobronchial metastatic disease. Thorax. 1982;37:362-365. https://doi.org/10.1136/thx.37.5.362

8. Dursun $A B$, Demirag $F$, Bayiz $H$, Sertkaya D. Endobronchial metastases: $A$ clinicopathological analysis. Respirology. 2005;10(4):510-514. https://doi.org/ 10.1111/j.1440-1843.2005.00731.x

9. Carlson V, Delclos L, Fletcher GH. Distant metastases in squamous-cell carcinoma of the uterine cervix. Radiology 1967;88:961-966. https://doi.org/10.1148/88.5.961

10. Heitmiller RF, Marasco WJ, Hruban RH, Marsh BR. Endobronchial metastasis. Jurg. 1993;106:537-542.

11. Baumgartner WA, Mark JB. Metastatic malignancies from distant sites to the tracheobronchial tree. J Thorac Cardiovasc Surg. 1980;79:499-503.

12. Katsimbri PP, Bamias AT, Froudarakis ME, Peponis IA, Constantopoulos SH, Pavlidis NA. Endobronchial metastases secondary to solid tumors; report of eight cases and review of the literature. Lung Cancer. 2000;28:163-170. https://doi. org/10.1016/S0169-5002(99)00134-8

13. Braman SS, Whitcomb ME. Endobronchial metastasis. Arch Intern Med. 1975;135:543-547. https://doi.org/10.1001/archinte.1975.00330040055008

14. Walboomers JM, Jacobs MV, Manos MM, et al. Human papillomavirus is a necessary cause of invasive cervical cancer worldwide. J Pathol. 1999;189:12. https://doi.org/10.1002/(SICI)1096-9896(199909)189:1<12::AID-PATH431> https://doi.

15. ZurHausen $\mathrm{H}$. Papillomavirus infections - A major cause of human cancers. Biochim Biophys Acta. 1996;1288(2):F55-F78. https://doi.org/10.1016/0304419X(96)00020-0

16. Negri G, Egarter-Vigl E, Kasal A, Romano F, Haitel A, Mian C. p16 ${ }^{\text {INK4a }}$ is a useful marker for the diagnosis of adenocarcinoma of the cervix uteri and its precursors: An immunohistochemical study with immunocytochemical correlations. Am J Surg Pathol. 2003;27(2):187-193. https://doi.org/10.1097/00000478-200302000Surg Path
00006

17. Lesnikova I, Lidang M, Hamilton-Dutoit S, Koch J. p16 as a diagnostic marker of cervical neoplasia: A tissue microarray study of 796 archival specimens. Diagn Pathol. 2009;4:22. https://doi.org/10.1186/1746-1596-4-22 